Check for updates

Cite this: RSC Adv., 2019, 9, 33976

\title{
Graphene chemiresistors modified with functionalized triphenylene for highly sensitive and selective detection of dimethyl methylphosphonate $\uparrow$
}

\author{
Yun-Tae Kim, ${ }^{a}$ Seongwoo Lee, ${ }^{b}$ Sanghwan Park ${ }^{a}$ and Chang Young Lee (D) *a
}

Graphene has attracted significant attention from researchers in recent years as a gas sensing material, because of its atom-thick 2-D structure, extremely high surface-to-volume ratio, and high carrier mobility. However, chemiresistive gas sensors based on graphene have a drawback of low sensitivity to organophosphates, including dimethyl methylphosphonate (DMMP), a simulant of the nerve agent sarin. In this study, we report the detection of $1.3 \mathrm{ppm}$ DMMP, the highest sensitivity reported to date, using graphene chemiresistors, by non-covalently functionalizing graphene with $N$-substituted triphenylene. The functionalized graphene sensor exhibits a two orders of magnitude higher response to DMMP than to other compounds. This high sensitivity and selectivity are attributed to the strong hydrogen bonding between DMMP and $N$-substituted triphenylene, as well as the hole-doping effect caused by triphenylene, which increases the binding affinity to the electron-donating DMMP. The proposed approach for simple functionalization of graphene with substituted triphenylene can potentially be employed in tuning the properties of other conjugated nanomaterials, such as carbon nanotubes and graphene nanoribbons, to detect various target analytes.

Received 7th September 2019
Accepted 17th October 2019

DOI: 10.1039/c9ra07186e

rsc.li/rsc-advances when detecting low-concentrations of analytes, such as chemical warfare agents (CWA).

The high sensitivity of nanomaterials is highly advantageous in detecting CWAs because CWAs are lethal even at low concentrations. The nerve agent sarin, for example, causes death within $10 \mathrm{~min}$ at $64 \mathrm{ppb} ;{ }^{11}$ various nanomaterials have been reported to detect the ppt-ppb level of dimethyl methylphosphonate (DMMP), ${ }^{11-13}$ a simulant of sarin. As for the graphene, graphene oxide (GO), and reduced graphene oxide ( $\mathrm{rGO}$ ), however, the lowest DMMP concentration detected was only 5-25 $\mathrm{ppm}^{14-17}$ even after functionalization with polypyrrole (PPy) ${ }^{15}$ single-stranded DNA, ${ }^{17}$ or $p$-phenylenediamine. ${ }^{14}$ Moreover, the carrier mobility in graphene decreases after the functionalization because of the scattering of charge carriers. ${ }^{17}$ Thus, such limitations of the graphene gas sensors must be addressed by employing a new functionalization approach that improves the sensitivity of graphene without altering its electrical properties.

In this study, we functionalize graphene with $N$-substituted triphenylenes obtained by palladium-catalyzed one-step synthesis ${ }^{18}$ to detect DMMP with high sensitivity and selectivity. The functionalized triphenylenes are easily soluble, processable, and applicable to graphene-based chemiresistive gas sensors. The electrical properties of graphene can be manipulated without degrading its material properties. ${ }^{18}$ The graphene sensor developed in this study exhibits a sensitivity of
${ }^{a}$ School of Energy and Chemical Engineering, Ulsan National Institute of Science and Technology (UNIST), Ulsan 44919, Republic of Korea. E-mail: cylee@unist.ac.kr ${ }^{b}$ School of Materials Science and Engineering, Ulsan National Institute of Science and Technology (UNIST), Ulsan 44919, Republic of Korea

$\dagger$ Electronic supplementary information (ESI) available. See DOI: 10.1039/c9ra07186e 
1.3 ppm to DMMP, which is the highest of all graphene-based chemiresistors reported till date. In addition, its response to DMMP is two orders of magnitude higher than that to other volatile organic compounds, indicating high selectivity of the functionalized graphene sensor.

\section{Materials and methods}

\section{Synthesis and transfer of graphene}

Graphene was synthesized on copper foil substrates $(25 \mu \mathrm{m}$, Alfa Aesar) via low-pressure chemical vapor deposition. The copper foils were annealed under a hydrogen atmosphere $\left(1000{ }^{\circ} \mathrm{C}, 30 \mathrm{~min}, 9 \mathrm{sccm}\right.$.) followed by graphene synthesis with a gas mixture of methane and hydrogen $\left(1000{ }^{\circ} \mathrm{C}, 30 \mathrm{~min}, 17\right.$ sccm for methane and $9 \mathrm{sccm}$ for hydrogen). The graphene on copper was spin-coated with poly (methyl methacrylate) (PMMA) and dried in an oven to remove the solvent. The copper foils were then etched out by ferric chloride solution. After copper etching, the PMMA-graphene layer was scooped out using clean water and thoroughly rinsed. It was then transferred onto the target substrate and the PMMA was removed using acetone.

\section{Fabrication of graphene gas sensor}

After transferring the graphene onto a $300 \mathrm{~nm}$-thick $\mathrm{SiO}_{2} / \mathrm{Si}$ substrate, the gold electrode pads $(10 / 70 \mathrm{~nm}$ of $\mathrm{Cr} / \mathrm{Au})$ for graphene were patterned using conventional photolithography. Subsequently, arrays of graphene microribbons were fabricated by photolithography and etched out by reactive ion etching (RIE) at $20 \mathrm{sccm}$ of $\mathrm{O}_{2}$ and $100 \mathrm{mTorr}$ for $3 \mathrm{~min}$, followed by the removal of photoresist in acetone. The width and length of the ribbons are $10 \mu \mathrm{m}$ and $200 \mu \mathrm{m}$, respectively, with the spacing between the ribbons being $10 \mu \mathrm{m}$. To avoid batch-to-batch variances, a single-batch graphene was cut into many small pieces of sensor devices $(1.2 \mathrm{~mm} \times 4.7 \mathrm{~mm})$ using a dicing saw.

\section{Raman spectroscopy}

Raman spectroscopy was performed using the WITec Alpha 300 system equipped with a $532 \mathrm{~nm}(2.33 \mathrm{eV})$ excitation laser. All measurements were performed with linear polarization, and using a $\times 50$ objective lens with a grating of 600 grooves per $\mathrm{mm}$.

\section{Graphene functionalization with substituted triphenylene}

The $N$-substituted triphenylenes were dissolved in $o$-dichlorobenzene (ODCB) at a concentration of $2 \times 10^{-3} \mathrm{M}$. A droplet of substituted triphenylene $(2.5 \mu \mathrm{l})$ was dropped onto the graphene gas sensor and the solvent was allowed to evaporate at room temperature.

\section{Gas sensing measurement}

The resistance changes were measured using a digital multimeter (Agilent, 34410A). The DMMP vapor $(10 \mathrm{ml})$ was injected at a flow rate of $\sim 3 \mathrm{ml} \mathrm{s}^{-1}$ by a syringe at room temperature. A disposable syringe was used at every injection to avoid ambiguity caused by residual DMMP in the syringe.

\section{Results and discussion}

$N$-substituted triphenylenes were synthesized via one-step reaction by palladium-catalyzed 2-fold $\mathrm{C}-\mathrm{H}$ arylation, as reported previously (Fig. S1†). ${ }^{18}$ The functionalized triphenylenes are stable and can thus be handled in air. A monolayer graphene was synthesized by chemical vapor deposition (CVD) on a copper foil, and transferred onto a silicon substrate with thermal oxides, using the poly(methyl methacrylate) (PMMA)-mediated transfer method. ${ }^{19}$ Furthermore, arrays of graphene microribbons were fabricated by photolithography and oxygen plasma etching, as shown in Fig. 1a. The width and length of the ribbons were $10 \mu \mathrm{m}$ and $200 \mu \mathrm{m}$, respectively, with a spacing of $10 \mu \mathrm{m}$ between the ribbons. We selected the microribbon structure of graphene, considering its enhanced sensitivity to target analytes because of the edge effects. ${ }^{20}$ Moreover, substituted triphenylenes can be potentially employed in tuning the properties of conjugated systems including graphene, via $\pi-\pi$ stacking. Fig. 1b illustrates the non-covalent functionalization of graphene microribbons using $N$-substituted triphenylene. The Raman spectra were collected from our CVD-grown graphene both before and after the functionalization, as shown in Fig. 1c. The average intensity ratio of the 2-D peak to G peak $\left(I_{2 \mathrm{D}} / I_{\mathrm{G}}\right)$ was 1.83 for the pristine graphene, which decreased to 1.65 after the functionalization (Fig. S2 $\dagger$ ). No D peak was observed in both pristine and functionalized graphene. The Raman spectra results indicate that the graphene used in this study is predominantly a monolayer with almost no defects, and the functionalization by substituted triphenylene is non-covalent.

To improve the response to DMMP vapor, we first tuned the electrical properties of graphene by heavy hole-doping. Reports
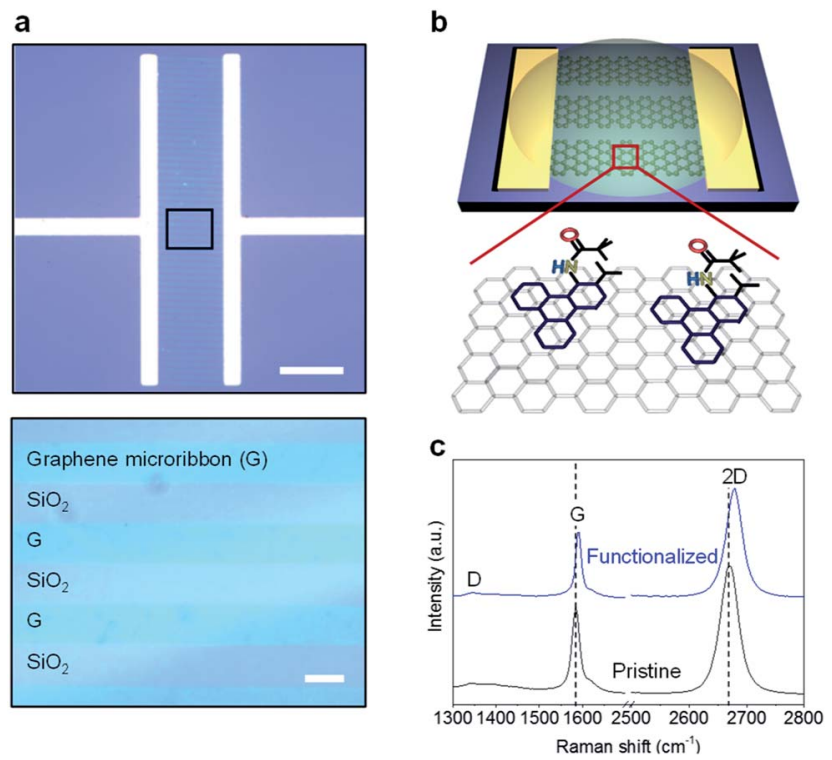

Fig. 1 Graphene microribbons as a chemiresistive gas sensor. (a) Images of graphene microribbon sensor (top) and zoomed-in image (bottom) of the box in (a). Scale bars: $200 \mu \mathrm{m}$ (top) and $10 \mu \mathrm{m}$ (bottom). (b) Schematic of the functionalized graphene with substituted triphenylene via $\pi-\pi$ stacking. (c) Raman spectra of graphene before and after functionalization. 
indicate that heavy-hole-doped and electro-deficient carbon nanomaterials increase the binding affinity and charge transfer of electron-donating molecules, such as $\mathrm{DMMP}^{11}$ and $\mathrm{NH}_{3}{ }^{21,22}$ The doping of graphene by triphenylene functionalization can be assessed using the $\mathrm{G}$ and 2-D peaks. ${ }^{23-28}$ Doped graphene exhibits an upshift in the position of the $\mathrm{G}$ peak $\left(\omega_{\mathrm{G}}\right)$ but a decreased full width at half maximum (FWHM) of G peak $\left(\Gamma_{\mathrm{G}}\right) \cdot{ }^{23-28}$ Fig. 2a plots $\Gamma_{\mathrm{G}}$ against $\omega_{\mathrm{G}}$ for our graphene before (black) and after (blue) the functionalization, which enhances the doping effect of triphenylene, as indicated by the shift of $\Gamma_{\mathrm{G}}$ and $\omega_{\mathrm{G}}$ in the direction of the dotted arrow. The type of doping can be identified by examining the 2-D peak position $\left(\omega_{2 \mathrm{D}}\right)$, which downshifts and sharpens upon n-doping. ${ }^{23-28}$ We plotted $\omega_{2 \mathrm{D}}$ against $\omega_{\mathrm{G}}$ both before (black) and after (blue) the triphenylene functionalization of our graphene, as shown in Fig. $2 b$. In the figure, the black circle at $\omega_{\mathrm{G}}=1581.6 \pm 1 \mathrm{~cm}^{-1}$ and $\omega_{2 \mathrm{D}}=$ $2678.6 \pm 1 \mathrm{~cm}^{-1}$ indicates the $\mathrm{G}$ and 2-D peak positions of an intrinsic graphene without any doping. ${ }^{24}$ Upon doping of the intrinsic graphene, the peak positions move toward the topright and bottom-right directions for $\mathrm{p}$ - and n-doping, respectively, as indicated by the dotted arrows. ${ }^{24}$ Prior to the triphenylene functionalization, the peak positions of our graphene overlaps the slightly p-doped region in this plot, because of the adsorption of oxygen and water molecules in ambient conditions, as well as the deposition of impurities during CVD.

Manipulating the properties of graphene without degrading its electrical properties is highly desired to realize a wide range

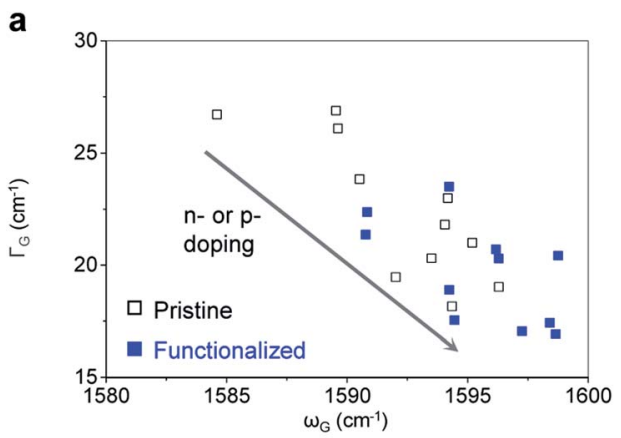

b

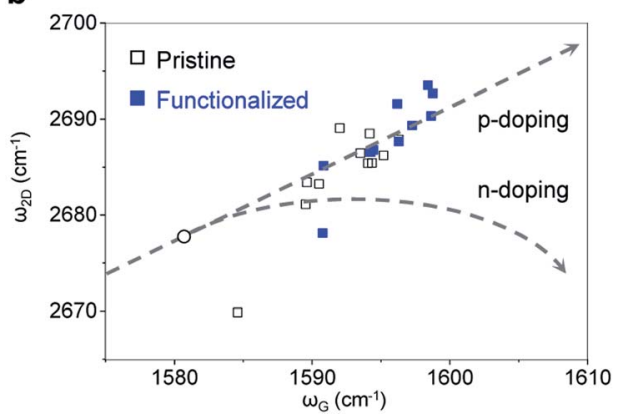

Fig. 2 Analysis of Raman peak parameters. (a) Full width at half maximum of $\mathrm{G}$ peak (FWHM, $\left.\Gamma_{\mathrm{G}}\right)$ versus peak position of $\mathrm{G}$ peak $\left(\omega_{\mathrm{G}}\right)$, showing the doping trend. (b) 2-D peak position ( $\omega_{2 D}$ ) versus $G$ peak position $\left(\omega_{\mathrm{G}}\right)$. The doping lines were adopted from [ref. 20]. The black circle indicates the $G$ and 2-D peak positions of an intrinsic graphene, where graphene has neither doping nor strain growth. ${ }^{29-31}$ After the functionalization, the graphene is further $\mathrm{p}$-doped. of applications of graphene. Previous studies have reported various dopants for graphene $;^{\mathbf{3 2 - 3 6}}$ however, there still remain a few challenges on developing dopants that can be processed and patterned easily. Our solution-processable p-type dopant has the potential to manipulate the electrical properties of graphene, which we demonstrate for gas sensing applications.

We measured in situ, the resistance change of graphene upon exposure to DMMP vapor before and after the functionalization with substituted triphenylene. Fig. 3a shows the resistance change of a graphene microribbon sensor upon exposure to DMMP vapor. Initially, the pristine graphene sensor was exposed to three pulses of $1.3 \mathrm{ppm}$ DMMP vapor, corresponding to the period colored in green. The responses indicated an almost complete recovery to the baseline within $50 \mathrm{~s}$, suggesting a complete desorption of DMMP from the graphene surface. Alongside recording the resistance, the sensor was simultaneously functionalized by drop-drying the triphenylene solution at $695 \mathrm{~s}$, from which point the graph is colored in blue. The resistance decreased sharply after the drop, and stabilized in $\sim 70 \mathrm{~s}$ upon evaporation of the solvent. The baseline resistance after stabilization permanently decreased from 527 to 500, which confirms a successful functionalization and pdoping of the graphene by triphenylene. The triphenylene functionalization increased the sensitivity of graphene to DMMP by 2.5 times (Fig. 3b), possibly because of the hydrogen bonding between DMMP and triphenylene. ${ }^{11,15}$ A rGO multilayer framework reported detection of 1 ppm DMMP,${ }^{37}$ but our result showing detection at $1.3 \mathrm{ppm}$ is the best among graphene sensors. In addition, the enhanced interaction with DMMP
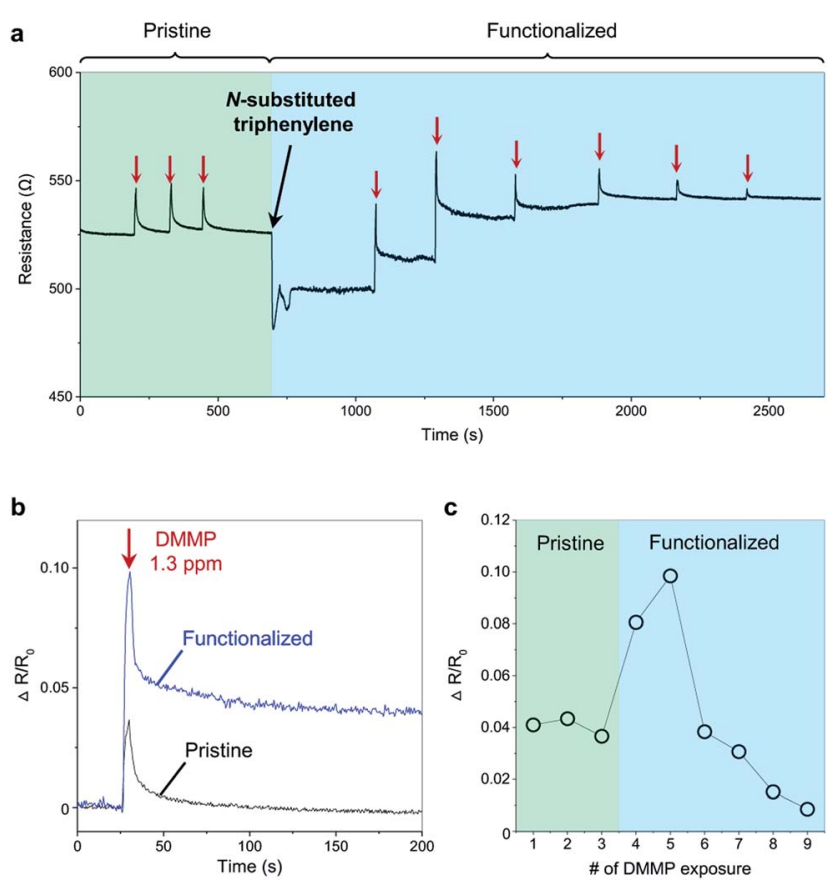

Fig. 3 Detecting DMMP vapor using the functionalized graphene sensor. (a) In situ graphene functionalization and DMMP sensing. All concentrations of DMMP were $1.3 \mathrm{ppm}$. (b) Typical responses to DMMP before and after functionalization. (c) Change in sensor response upon repeated exposure to 1.3 ppm DMMP vapor. 
made the response partially irreversible, causing the sensor response to gradually decrease upon repeated exposure of DMMP, as shown in Fig. 3c. Once the binding sites on the surface of graphene were occupied by the analytes, the sensor response became very weak even at high concentrations of $\sim 1300$ ppm (Fig. S3†). The in situ measurement presented in this study enables a clear understanding of the coating effect using an identical sensor, eliminating the need to consider the sensor-to-sensor variation of performance.

As discussed above, the pristine graphene that is initially $\mathrm{p}$ type becomes further p-doped by triphenylene. Upon irreversible adsorption of the $n$-doping DMMP, ${ }^{15,38,39}$ the functionalized graphene returns to the doping state of the pristine graphene, which is corroborated by the Raman peak parameters, such as $I_{2 \mathrm{D}} / I_{\mathrm{G}}, \omega_{\mathrm{G}}, \omega_{2 \mathrm{D}}$, and $\Gamma_{\mathrm{G}}$ (Fig. S4 $\dagger$ ).

To evaluate the selectivity of our graphene toward the DMMP, we compared the sensing responses for various interfering volatile organic compounds (VOC), including 1,2dichlorobenzene, toluene, 1,4-dichlorobutane, acetone, methanol, and water. As shown in Fig. 4, the DMMP elicits two orders of magnitude higher response than the others, which can be attributed to the formation of a strong hydrogen bonding with $\mathrm{N}$-substituted triphenylene, because of additional acceptors $\left(-\mathrm{OCH}_{3}\right)$ and the relatively high polarity (3.62 D) of DMMP. ${ }^{\mathbf{1 2 , 4 0}}$ In addition, the graphene further p-doped by the functionalization has greater binding affinity toward electron-donating DMMP. ${ }^{11,21,22}$ The graphene gas sensor functionalized with $N$-substituted triphenylene has high sensitivity and selectivity toward DMMP, making for an excellent candidate for ultrasensitive and selective detection of DMMP. Note that the large variation of $\Delta R / R_{0}$ values in the DMMP responses is attributed to the non-uniform properties of pristine graphene, caused by the wrinkles, islands of multilayer graphene, and the electron-hole puddles on $\mathrm{a} \mathrm{SiO}_{2}$ substrate. ${ }^{23}$ Non-uniform coverage of triphenylene molecules, caused by the ring-stains ${ }^{\mathbf{4 1 4}}$ formed during drop-casting, further increases the variation of $\Delta R / R_{0}$. The sensor-to-sensor variation may be reduced by the use of a single-layer graphene with high crystallinity and fabrication of the sensor on a substrate that lowers the charge inhomogeneity. ${ }^{31,43}$

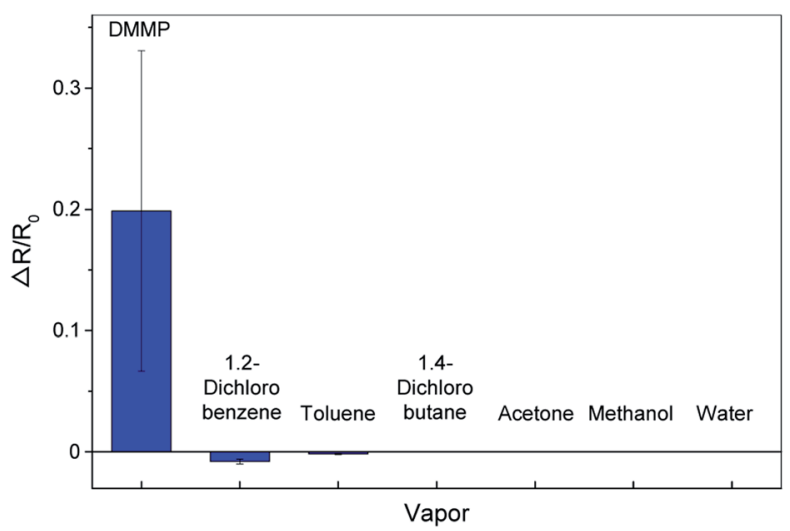

Fig. 4 Response of graphene gas sensors to DMMP and interfering volatile organic compounds at the same concentration ( $\sim 1 \mathrm{ppm})$.

\section{Conclusions}

We demonstrated highly selective and sensitive graphene chemiresistors to detect DMMP, a nerve agent simulant, via non-covalent functionalization of graphene with substituted triphenylenes by simple solution processing. This functionalization further p-dopes the graphene without degrading its electrical properties, which helps detect the electron-donating molecules, including DMMP. Our sensor detects DMMP with the highest sensitivity (1.3 ppm) reported till date, of all present graphene chemiresistors. Furthermore, its response to DMMP is two orders of magnitude greater than that to other VOCs because of the hydrogen bonding between DMMP and $\mathrm{N}$ substituted triphenylene. Being based on $\pi-\pi$ stacking, the non-covalent functionalization of graphene with substituted triphenylene can be employed in tuning the properties of other conjugated carbon nanomaterials, such as carbon nanotubes and graphene nanoribbons.

\section{Conflicts of interest}

There are no conflicts to declare.

\section{Acknowledgements}

Authors thank Prof. Sung You Hong at UNIST for providing the functionalized triphenylenes.

\section{Notes and references}

1 C. Y. Lee and M. S. Strano, J. Am. Chem. Soc., 2008, 130, 17661773.

2 V. Schroeder, S. Savagatrup, M. He, S. B. Ling and T. M. Swager, Chem. Rev., 2019, 119, 599-663.

3 D. R. Kauffman and A. Star, Angew. Chem., Int. Ed., 2008, 47, 6550-6570.

4 H. Bai and G. Q. Shi, Sensors, 2007, 7, 267-307.

5 S. J. Park, C. S. Park and H. Yoon, Polymers, 2017, 9, 155.

6 N. Joshi, T. Hayasaka, Y. M. Liu, H. L. Liu, O. N. Oliveira and

L. W. Lin, Microchim. Acta, 2018, 185.

7 A. Rehman and X. Q. Zeng, RSC Adv., 2015, 5, 58371-58392.

8 W. J. Yuan and G. Q. Shi, J. Mater. Chem. A, 2013, 1, 1007810091.

9 S. S. Varghese, S. Lonkar, K. K. Singh, S. Swaminathan and A. Abdala, Sens. Actuators, B, 2015, 218, 160-183.

10 T. Wang, D. Huang, Z. Yang, S. S. Xu, G. L. He, X. L. Li, N. T. Hu, G. L. Yin, D. N. He and L. Y. Zhang, Nano-Micro Lett., 2016, 8, 95-119.

11 L. M. Wei, D. W. Shi, P. Y. Ye, Z. Q. Dai, H. Y. Chen, C. X. Chen, J. Wang, L. Y. Zhang, D. Xu, Z. Wang and Y. F. Zhang, Nanotechnology, 2011, 22, 425501.

12 S. Cho, O. S. Kwon, S. A. You and J. Jang, J. Mater. Chem. A, 2013, 1, 5679-5688.

13 J. Jun, J. S. Lee, D. H. Shin, J. Oh, W. Kim, W. Na and J. Jang, J. Mater. Chem. A, 2017, 5, 17335-17340. 
14 N. T. Hu, Y. Y. Wang, J. Chai, R. G. Gao, Z. Yang, E. S. W. Kong and Y. F. Zhang, Sens. Actuators, B, 2012, 163, 107-114.

15 J. Park, J. Kim, K. Kim, S. Y. Kim, W. H. Cheong, K. Park, J. H. Song, G. Namgoong, J. J. Kim, J. Heo, F. Bien and J. U. Park, Nanoscale, 2016, 8, 10591-10597.

16 T. Alizadeh and L. H. Soltani, Sens. Actuators, B, 2016, 234, 361-370.

17 Y. Lu, B. R. Goldsmith, N. J. Kybert and A. T. C. Johnson, Appl. Phys. Lett., 2010, 97, 083107.

18 B. P. Mathew, H. J. Yang, J. Kim, J. B. Lee, Y. T. Kim, S. Lee, C. Y. Lee, W. Choe, K. Myung, J. U. Park and S. Y. Hong, Angew. Chem., Int. Ed., 2017, 56, 5007-5011.

19 A. Reina, X. T. Jia, J. Ho, D. Nezich, H. B. Son, V. Bulovic, M. S. Dresselhaus and J. Kong, Nano Lett., 2009, 9, 30-35.

20 A. Salehi-Khojin, D. Estrada, K. Y. Lin, M. H. Bae, F. Xiong, E. Pop and R. I. Masel, Adv. Mater., 2012, 24, 53-57.

21 Q. F. Pengfei, O. Vermesh, M. Grecu, A. Javey, O. Wang, H. J. Dai, S. Peng and K. J. Cho, Nano Lett., 2003, 3, 347-351. 22 S. M. M. Zanjani, M. M. Sadeghi, M. Holt, S. F. Chowdhury, L. Tao and D. Akinwande, Appl. Phys. Lett., 2016, 108, 033106.

23 Q. H. Wang, Z. Jin, K. K. Kim, A. J. Hilmer, G. L. C. Paulus, C. J. Shih, M. H. Ham, J. D. Sanchez-Yamagishi, K. Watanabe, T. Taniguchi, J. Kong, P. Jarillo-Herrero and M. S. Strano, Nat. Chem., 2012, 4, 724-732.

24 L. Banszerus, H. Janssen, M. Otto, A. Epping, T. Taniguchi, K. Watanabe, B. Beschoten, D. Neumaier and C. Stampfer, 2D Mater., 2017, 4.

25 S. Pisana, M. Lazzeri, C. Casiraghi, K. S. Novoselov, A. K. Geim, A. C. Ferrari and F. Mauri, Nat. Mater., 2007, 6, 198-201.

26 C. Stampfer, F. Molitor, D. Graf, K. Ensslin, A. Jungen, C. Hierold and L. Wirtz, Appl. Phys. Lett., 2007, 91.

27 M. Kalbac, A. Reina-Cecco, H. Farhat, J. Kong, L. Kavan and M. S. Dresselhaus, ACS Nano, 2010, 4, 6055-6063.

28 A. Das, S. Pisana, B. Chakraborty, S. Piscanec, S. K. Saha, U. V. Waghmare, K. S. Novoselov, H. R. Krishnamurthy,
A. K. Geim, A. C. Ferrari and A. K. Sood, Nat. Nanotechnol., 2008, 3, 210-215.

29 P. Wei, N. Liu, H. R. Lee, E. Adijanto, L. J. Ci, B. D. Naab, J. Q. Zhong, J. Park, W. Chen, Y. Cui and Z. A. Bao, Nano Lett., 2013, 13, 1890-1897.

30 S. Y. Chen, P. H. Ho, R. J. Shiue, C. W. Chen and W. H. Wang, Nano Lett., 2012, 12, 964-969.

31 M. Lafkioti, B. Krauss, T. Lohmann, U. Zschieschang, H. Klauk, K. von Klitzing and J. H. Smet, Nano Lett., 2010, 10, 1149-1153.

32 S. Huh, J. Park, K. S. Kim, B. H. Hong and S. Bin Kim, ACS Nano, 2011, 5, 3639-3644.

33 K. S. Han, P. Y. Kalode, Y. E. K. Lee, H. Kim, L. Lee and M. M. Sung, Nanoscale, 2016, 8, 5000-5005.

34 S. Ji, S. J. Kim, W. Song, S. Myung, J. Heo, J. Lim, K. S. An and S. S. Lee, $R S C A d v ., 2016,6$, 19372-19376.

35 J. Baltazar, H. Sojoudi, S. A. Paniagua, S. Y. Zhang, R. A. Lawson, S. R. Marder, S. Graham, L. M. Tolbert and C. L. Henderson, Adv. Funct. Mater., 2014, 24, 5147-5156.

36 Z. Arefinia and A. Asgari, J. Appl. Phys., 2014, 115, 194506.

37 Y. Y. Wang, M. Yang, W. X. Liu, L. Dong, D. Chen and C. S. Peng, J. Mater. Chem. C, 2019, 7, 9248-9256.

38 C. Y. Lee, R. Sharma, A. D. Radadia, R. I. Masel and M. S. Strano, Angew. Chem., Int. Ed., 2008, 47, 5018-5021.

39 J. P. Novak, E. S. Snow, E. J. Houser, D. Park, J. L. Stepnowski and R. A. McGill, Appl. Phys. Lett., 2003, 83, 4026-4028.

40 K. Kim, O. G. Tsay, D. A. Atwood and D. G. Churchill, Chem. Rev., 2011, 111, 5345-5403.

41 R. D. Deegan, O. Bakajin, T. F. Dupont, G. Huber, S. R. Nagel and T. A. Witten, Nature, 1997, 389, 827-829.

42 D. Mampallil and H. B. Eral, Adv. Colloid Interface Sci., 2018, 252, 38-54.

43 C. R. Dean, A. F. Young, I. Meric, C. Lee, L. Wang, S. Sorgenfrei, K. Watanabe, T. Taniguchi, P. Kim, K. L. Shepard and J. Hone, Nat. Nanotechnol., 2010, 5, 722726. 